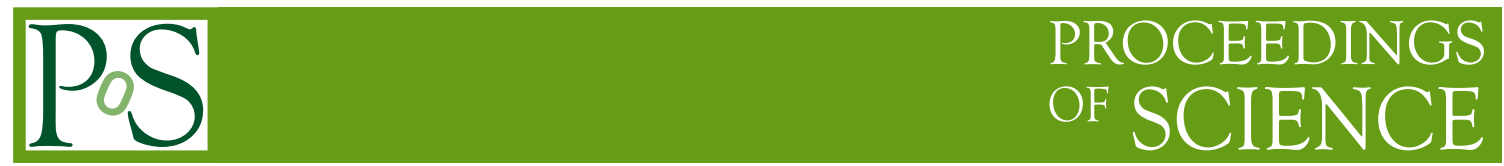

\title{
Topics in baryon chiral perturbation theory
}

\section{Ulf-G. Meißner}

Helmholtz-Institut für Strahlen- und Kernphysik and Bether Center for Theoretical Physics Universität Bonn, D-53115 Bonn, Germany

and

Institut für Kernphysik (IKP-3) and Jülich Center for Hadron Physics D-52425 Jülich, Germany

E-mail: meissner@itkp.uni-bonn.de

Baryon chiral perturbation theory can be used as a tool to analyze properties of excited baryons in lattice QCD. I discuss some recent developments in this cross-disciplinary field.

8th Conference Quark Confinement and the Hadron Spectrum

September 1-6, 2008

Mainz. Germany 


\section{Introduction}

Baryon chiral perturbation theory is the tool to analyse the strictures of the spontaneously and explicitly broken chiral symmetry of QCD (for systems with baryon number $B \geq 1$ ). There are many recent developments, from which I just list a few. Covariant formulations have been developed that allow to deal with the additional large mass scale in a manifestly Lorentz-inavriant manner like Infrared Regularization (IR) at Bern, the Extendend-On-Mass-Scheme at Mainz or the modified IR approach at Munich. Within these schemes and also using the older heavy baryon approach, quite a few successfull calculations at one loop oder have been performed, but of course some open issues remain to be clarified, like e.g. the puzzle related to the pion electroproduction data $p\left(\gamma^{*}, \pi^{0}\right) p$ at low photon virtualities form MAMI-B. There are also a few attempts of two-loop calculations (for the nucleon axial-vector coupling $g_{A}$ and the nucleon mass $m_{N}$ ). Furthermore, extensions of baryon CHPT have been developed to include the lowest-lying resonances like the $\Delta(1232)$ and the $N^{\star}(1440)$ (as will be discussed below) but also some work on effective theories including baryons and vector mesons have been performed. Another important field of high current interest are the so-called unitary extensions for the three-flavor case, in which the strict perturbative expansion is given up in favor of a resummation of certain classes of s-channel diagrams. This allows for studies of resonances, in particular concerning the nature of the $\Lambda(1405)$ or $S_{11}(1535)$ and their influene on kaon(eta)-nucleon scattering or electromagnetic meson production. Most of these topics have been nicely reviewed by Bernard [1]. Furthermore, baryon CHPT has become an indispensable tool for lattice QCD practioneers (chiral extrapolations and finite volume effects), and it thus appears appropriate to discuss some very recent results in this field in this talk (for reviews, see e.g. [1,2]). More precisely, I will focus on the extraction of the properties of baryon resonances made of the light $u, d, s$ quarks from lattice data, since this field has gained substantial interest recently in the lattice community (see e.g. $[3,4,5]$ ) and is also in the focus of the experimental programs at Jefferson Lab, ELSA at Bonn and COSY at Jülich.

\section{The $\Delta(1232)$ on the lattice}

Arguably the least understood part of the Standard Model is the spectrum of the strongly interacting particles. The only ab initio method to calculate the spectrum from the Lagarangian of QCD is based on its lattice formulation (space-time discretization) - from now called lattice QCD. In general, the extraction of the properties of the excited states from the lattice data is a more complicated enterprise as compared to the ground-state hadrons. The reason is that the excited states are unstable and, strictly speaking, can not be put in correspondence to a single isolated level in the discrete spectrum measured in lattice simulations. A standard procedure proposed by Lüscher (and others) consists in placing the system into a finite cubic box of a size $L$ and studying the response of the spectrum on the change of $L$. It can be shown that the dependence of the energy levels on $L$ is dictated solely by the scattering phase shift in the infinite volume. Consequently, the method is capable of extracting the phase shift from the lattice data that also determines the position and the width of the resonances (see, e.g. [6, 7] for recent calculations of the $\rho$-meson). Let us concentrate from here on on the most important (and lowest lying) baryon resonance, the $\Delta(1232)$. In actual calculations on the lattice the quark masses do not usually coincide with physical quark 

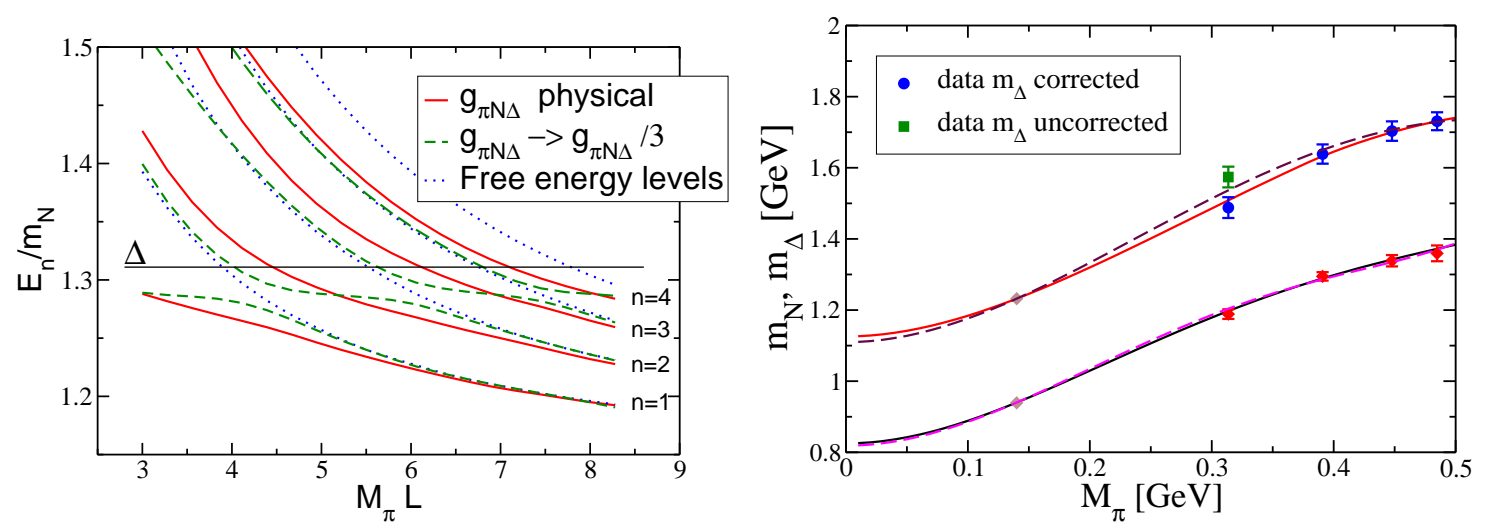

Figure 1: Left panel: Energy levels of the $\Delta(1232)$ in a box calculated to third order in the SSE (solid lines). The dashed lines correspond to a reduction of the $\Delta$ width by a factor 3 and the dotted ones to the free energy levels. Right panel: Chiral extrapolation of the $\Delta^{++}$(upper curves) and nucleon (lower curves) masses for the ETM data. Note that withoiut the finite volume correction on the lowest quark mass point for the $\Delta^{++}$ one observes the bump mentioned in the text.

masses. This qualitatively changes the picture since, if the quark mass is large enough, the $\Delta$ does not decay and can be extracted by the methods applicable in case of the stable particles. Reducing the quark mass, a value is achieved when the $\Delta$ starts to decay into a pion and a nucleon. The spectrum becomes strongly volume-dependent and Lüscher's method has to be applied to extract the parameters of the resonance - the mass and the width. It can be seen that the large finitevolume correction affects the curve that describes the quark-mass dependence of the resonance spectrum. Above the decay threshold, the finite-volume corrections to the spectrum are small and can be neglected in the first approximation. However, for those values of the quark masses which correspond to an unstable $\Delta$, finite-volume corrections become large and should be taken into account. Moreover, merely making the volume larger does not suffice in the case of an unstable state. Due to the large corrections, the finite volume data develop a "bump" below threshold, which is visible at smaller volumes and which can not be described by using the formulae for the quark mass dependence in the infinite volume. From the above discussion it is clear that, order to be able to include all available lattice data for large as well as small quark masses in the analysis, one needs to provide a simultaneous explicit parameterization of the lattice QCD spectrum in terms of both the quark mass $\hat{m}$ and the box size $L$. This goal can be achieved by invoking the chiral effective field theory with explicit spin-3/2 degrees of freedom $[8,9]$ in a finite volume. The first attempt in this direction was made in Ref. [10], where we have performed the calculations of the finite-volume energy spectrum at third order in the so-called small scale expansion (SSE) ${ }^{1}$. In that paper, it was shown that although the the avoided level crossing that appears for narrow resoances is washed out in case of the $\Delta$ due to its large width (see Fig. 1), its parameters can be determined from the lowest energy levels. More precisely, one fits the mass (width) of the $\Delta$ to the gradient of the lowest energy level (to the energy level difference $E_{2}-E_{1}$ ). We have recently extended this calculation to fourth order in the SSE and analyzed in detail the finite volume corrections in case

\footnotetext{
${ }^{1}$ In the SSE, the nucleon- $\Delta$ mass splitting is counted as a small parameter together with the external momenta and the pion mass.
} 


\begin{tabular}{|c|c|c|c|c|c|c|}
\hline$L(\mathrm{fm})$ & $M_{\pi}$ & $M_{N}$ & $E_{\Delta^{++,-}}$ & $E_{\Delta^{+, 0}}$ & $\delta E_{\Delta^{++,-}}$ & $\delta E_{\Delta^{+}, 0}$ \\
\hline \hline 2.1 & $314 \pm 2.4$ & $1189 \pm 14$ & $1574 \pm 29$ & $1609 \pm 40$ & -90 & -129 \\
\hline 2.7 & $309 \pm 1.9$ & $1177 \pm 13$ & $1523 \pm 23$ & $1523 \pm 34$ & -39 & -43 \\
\hline
\end{tabular}

Table 1: Meson and baryon masses for two different values of the box size $L$ (the data are taken from Table II of Ref. [12] (central values only) and correspond to the choice SS of the interpolating field). The last two columns correspond to the finite-volume corrections to the energy levels. All masses are given in MeV.

of an unstable $\Delta$ [11]. This was in particular spurred by the "kinky behaviour" of the mass of the $\Delta^{++}$as function of the pion mass in the data from the ETM collaboration [12] (see the right panel in Fig. 1). To understand this finite volume effect when the $\Delta$ becomes unstable, consider the $\Delta$ mass, which symbolically can be obtained from the equation

$$
m_{\Delta}=E+f\left(E, m_{N}, M_{\pi}\right) \bar{Z}_{00}\left(1 ; q^{2}\right), \quad \bar{Z}_{00}\left(1 ; q^{2}\right) \doteq Z_{00}\left(1 ; q^{2}\right)-\left.Z_{00}\left(1 ; q^{2}\right)\right|_{L \rightarrow \infty},
$$

with $Z_{00}\left(s ; q^{2}\right)=\sum_{n \in Z^{3}}\left(n^{2}-q^{2}\right)^{-s}, q=p L / 2 \pi, p=\lambda^{1 / 2}\left(E^{2}, m_{N}^{2}, M_{\pi}^{2}\right) / 2 E$ and the precise form of the function $f\left(E, m_{N}, M_{\pi}\right)$ is given in [11]. For $q^{2}<0$, the $\Delta$ is stable and the finite volume corrections are exponentially small. However, for an instable $\Delta\left(q^{2}>0\right)$, the finite volume corrections are sizeable. This is due to the fact that the function $q^{2} \bar{Z}_{00}\left(1 ; q^{2}\right)$ has a cusp, proportional to $q^{3}$, at threshold $q^{2}=0$. Moreover, its value in the limit $q^{2} \rightarrow 0$ is different from zero. This finite volume effect can now be calculated (for details, see [11]). In Table 1 the results for the finite-volume corrections to the lowest data point are given, see also Fig 1, where this effect is clearly visible. The results for the $\Delta^{+, 0}$ are presented just for the visualization of the artefacts due to the finite lattice size (in the twisted mass formualtion, the neutral pion mass is much lighter than the charged pion mass, inducing some large unphysical isospin breaking at finite lattice spacing). As can also seen from Fig 1, accounting for this finite volume effect allows for a very smooth and sensitive fit to the nucleon and the $\Delta$ mass. We remark that an analysis of the nucleon and $\Delta$ masses in twsted mass CHPT, was given in Ref. [13] and 'baryon masses at $O\left(a^{2}\right)$ in CHPT (including the $\Delta$ ) were considered in [14].

In a recent paper we have re-addressed the problem of the extraction of the $\Delta$ chracteristics within non-relativistic effective field theory (NR EFT) in a finite volume, which enables one to carry out the analysis in a more general way [15]. The equation that determines the location of the eigenvalues of the Hamiltonian in this framework coincides with Lüscher's formula. In order to facilitate the analysis, we further define a so-called probability distribution, which is constructed from the volume-dependent energies. Using Lüscher's formula, it can be shown that - to a good approximation - the probability distribution $W(p)$ can be expressed via the scattering phase

$$
W(p)=\frac{C}{p} \sum_{n=1}^{N}\left(\frac{\sqrt{4 \pi(\pi n-\delta(p))}}{p}+\frac{2 \pi \delta^{\prime}(p)}{\sqrt{4 \pi(\pi n-\delta(p))}}\right)
$$

where $\delta(p)$ denotes the scattering phase, $N$ is the number of the energy levels analyzed and $C$ denotes a normalization constant. Below we restrict ourselves to the analysis of the lowest state, putting $N=1$. The central observation is that the probability distribution in the vicinity of a resonance behaves much like the infinite-volume scattering cross section: it peaks at the resonance 

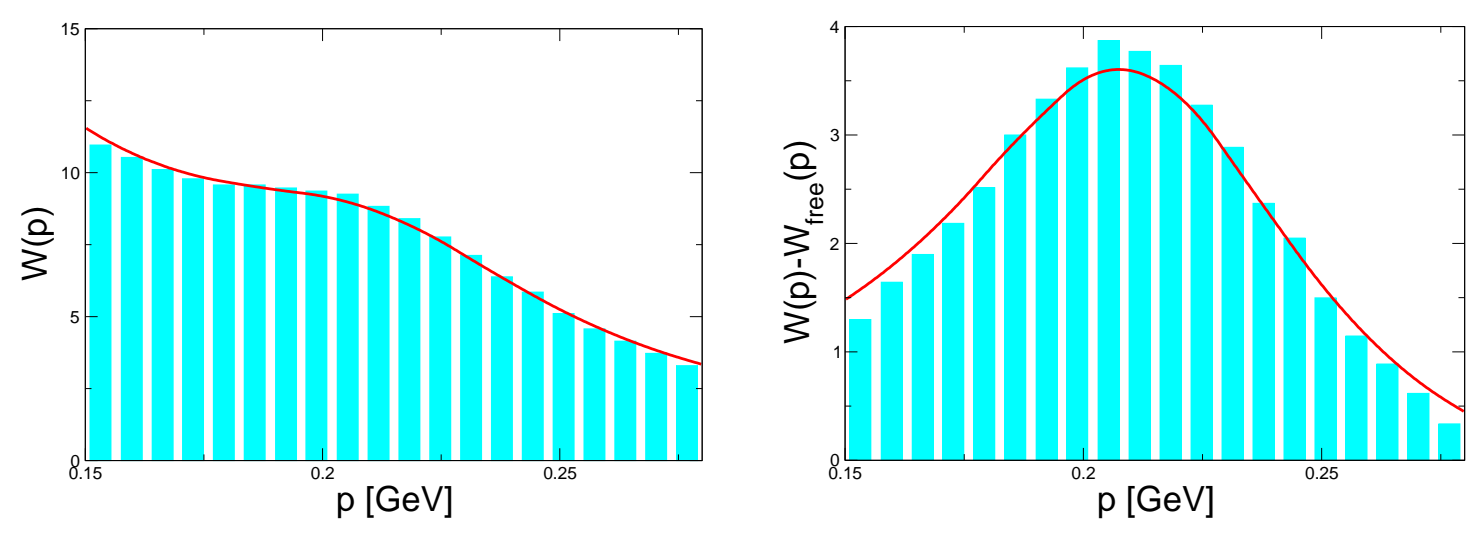

Figure 2: Unsubtracted (left panel) and subtracted (right panel) probability distributions. Only the lowest energy level has been included in the analysis $(N=1)$. The solid lines correspond to the prediction made by using Lüscher's formula. A clear resonance-like structure is observed in the subtracted distribution.

energy. The peak has approximately a Breit-Wigner shape, with the same width as the original resonance. In Ref. [15] it was shown that in case of a wide resonance, when the avoided level crossing is washed out, one still observes a clear resonance structure in the probability distribution after subtracting the background corresponding to the free motion of the decay products, corresponding to the free $\pi N$ pairs with $\delta(p)=0$. This is shown in Fig. 2 using pseudodata in the $P_{33}$ partial wave constructed from the VPI/GWU data base. This result unanimously supports the conclusion of Ref. [10]: the extraction of both the energy and width of the $\Delta$-resonance from the volume-dependent spectrum by using Lüscher's formula is feasible. Note also that this goal can be achieved even by fitting to the data for the lowest energy level alone. For more detailed on the method and its application, the reader is referred to Ref. [15]. We remark further that this method has recently been applied to a model Hamiltonian that has one narrow even-parity and one broad odd-parity resoance - both of which can be reproduced correctly, see Ref. [5].

\section{A method to extract the $K^{-} p$ scattering length from the finite-volume spectrum}

The antikaon-nucleon scattering amplitude is of fundamental importance in nuclear, particle and astrophysics. In particular, the $\bar{K} N$ system at threshold provides an interesting testing ground of the chiral dynamics of QCD with strange quarks due to the $\Lambda(1405)$ resonance just below the scattering threshold. In fact, experimental information on the $K^{-} p$ scattering length from scattering data and kaonic hydrogen level shifts is contradictory, as first pointed out in Ref. [16]. It would therefore be most welcome to have another tool at hand that would allow one to calculate this fundamental quantity. For the extraction of the $\bar{K} N$ scattering length, a generalization of the standard Lüscher scheme is required since there is a strong channel coupling between $\bar{K} N$ and $\Sigma \pi$, the latter channel having its threshold about $100 \mathrm{MeV}$ below the opening of the $\bar{K} N$ one. In addition, the appearance of the $\Lambda(1405)$ just between these two thresholds further complicates the picture. Such a scheme was recently proposed in [17]. In the large volumes required by the Lüscher formula, the characteristic momenta proportional to $L^{-1}$ are small. Therefore, the dynamics of a system can be described by non-relativistic effective field theory (NR EFT). For definiteness, we 
assume isospin conservation and restrict ourselves to the channel with total isospin $I=0$. Within dimensionally regularized NR EFT, the $S$-wave $T$-matrix in the infinite volume is the solution of a coupled-channel Lippmann-Schwinger equation (note that coupled channel dynamics in a finite box based on quantum mechanics was considered earlier in [18] and references therein)

$$
\begin{aligned}
& T_{11}=H_{11}+H_{11} i q_{1} T_{11}+H_{12} i q_{2} T_{21}, \\
& T_{21}=H_{21}+H_{21} i q_{1} T_{11}+H_{22} i q_{2} T_{21},
\end{aligned}
$$

where the channel number 1 refers to $\bar{K} N$ and 2 to $\Sigma \pi$ with total isospin $I=0$. The resonance $\Lambda(1405)$ is located between the two thresholds, on the second Riemann sheet, close to the real axis. These thresholds are $s_{t}=\left(m_{N}+M_{K}\right)^{2}, s_{t}^{\prime}=\left(m_{\Sigma}+M_{\pi}\right)^{2}$, with $q_{1}=\lambda^{1 / 2}\left(s, m_{N}^{2}, M_{K}^{2}\right) /(2 \sqrt{s})$, $q_{2}=\lambda^{1 / 2}\left(s, m_{\Sigma}^{2}, M_{\pi}^{2}\right) /(2 \sqrt{s})$ and $\lambda(x, y, z)$ stands for the Källen function. The quantities $H_{i j}(s)$ denote the driving potential in the corresponding channel. The $\bar{K} N$ scattering length is related to the amplitude $T_{11}$ at $s=s_{t}$ via

$$
a_{11} \equiv T_{11}\left(s_{t}\right)=H_{11}\left(s_{t}\right)+\frac{i q_{2}\left(s_{t}\right)\left(H_{12}\left(s_{t}\right)\right)^{2}}{1-i q_{2}\left(s_{t}\right) H_{22}\left(s_{t}\right)} .
$$

Thus, to pin down its complex value, we need to determine the three real quantities $H_{11}, H_{12}, H_{22}$ at $s=s_{t}$ appearing in Eq. (3.2). We now consider the same problem in a finite volume. The rotational symmetry is broken to a cubic symmetry so that the infinite volume version of the LS equation Eq. (3.1) takes the form (we consider only $S$-waves here, neglecting the small mixing to higher partial waves)

$$
\begin{aligned}
& T_{11}=H_{11}-\frac{2}{\sqrt{\pi} L} Z_{00}\left(1 ; k_{1}^{2}\right) H_{11} T_{11}-\frac{2}{\sqrt{\pi} L} Z_{00}\left(1 ; k_{2}^{2}\right) H_{12} T_{21}, \\
& T_{21}=H_{21}-\frac{2}{\sqrt{\pi} L} Z_{00}\left(1 ; k_{1}^{2}\right) H_{21} T_{11}-\frac{2}{\sqrt{\pi} L} Z_{00}\left(1 ; k_{2}^{2}\right) H_{22} T_{21},
\end{aligned}
$$

with $k_{1}^{2}\left(L^{2} / 4 \pi^{2}\right) q_{1}^{2}, k_{2}^{2}=\left(L^{2} / 4 \pi^{2}\right) q_{2}^{2}$, and

$$
Z_{00}\left(1 ; k^{2}\right)=\frac{1}{\sqrt{4 \pi}} \lim _{r \rightarrow 1} \sum_{\vec{n} \in Z^{3}} \frac{1}{\left(\vec{n}^{2}-k^{2}\right)^{r}}
$$

Here, we have neglected the terms that vanish exponentially at a large $L$. The secular equation that determines the spectrum can be brought into the form

$$
\begin{aligned}
& 1 \frac{2}{\sqrt{\pi} L} Z_{00}\left(1 ; k_{2}^{2}\right) F(s, L)=0 \\
& F(s, L)=\left[H_{22}-\frac{2}{\sqrt{\pi} L} Z_{00}\left(1 ; k_{1}^{2}\right)\left(H_{11} H_{22}-H_{12}^{2}\right)\right]\left[1-\frac{2}{\sqrt{\pi} L} Z_{00}\left(1 ; k_{1}^{2}\right) H_{11}\right]^{-1}
\end{aligned}
$$

This is rewritten as

$$
\delta(s, L)=-\phi\left(k_{2}\right)+n \pi, \quad \phi\left(k_{2}\right)=-\arctan \frac{\pi^{3 / 2} k_{2}}{Z_{00}\left(1 ; k_{2}^{2}\right)}, \quad n=0,1,2, \ldots,
$$




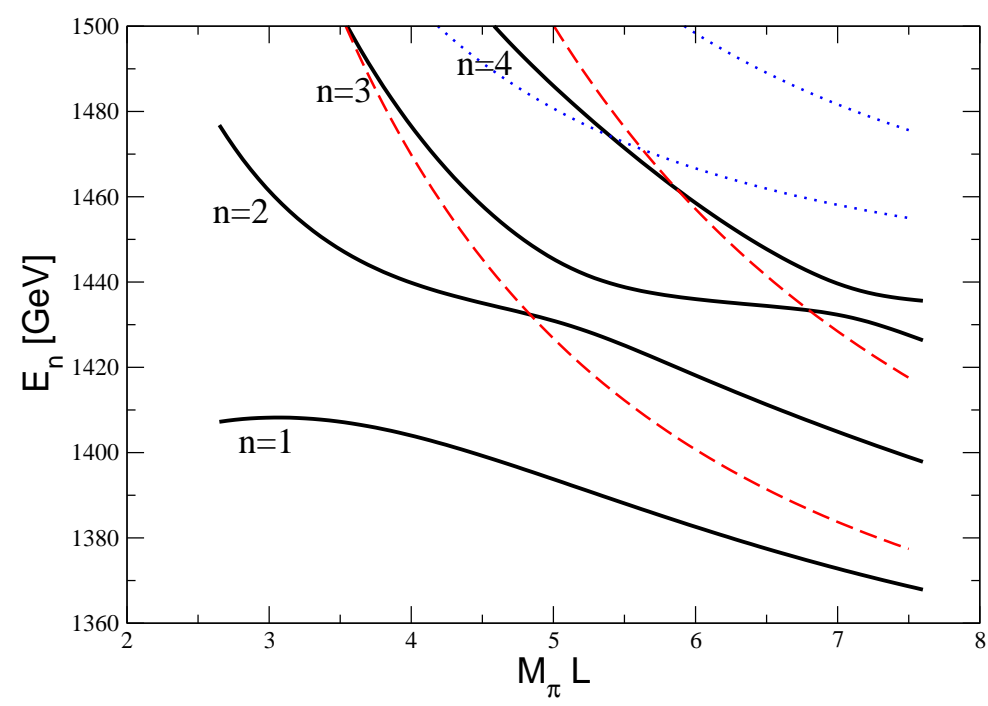

Figure 3: Energy levels for the two-channel model with an explicit $\Lambda(1405)$ resonance in the finite volume. The avoided level crossing which is observed at energies between $1430 \mathrm{MeV}$ and $1440 \mathrm{MeV}$ is not related to the physical resonance in the infinite volume but reflects the presence of the $\bar{K} N$ threshold. For comparison, we plot the energy levels for the non-interacting two-particle systems $\pi \Sigma$ (dashed lines) and $\bar{K} N$ (dotted lines).

with

$$
\tan \delta(s, L)=q_{2}(s) F(s, L) .
$$

$\delta(s, L)$ is called the pseudophase. The dependence of the pseudophase on $s$ and $L$ is very different from that of the usual scattering phase. Namely, the elastic phase extracted from the lattice data by using Lüscher's formula is independent of the volume modulo terms that exponentially vanish at a large $L$. Further, the energies where the phase passes through $\pi / 2$ lie close to the real resonance locations. In contrast to this, the pseudophase contains terms which are only power suppressed at a large $L$. Moreover, it contains the tower of resonances which are not related to the dynamics of the system in the infinite volume and merely reflect the existence of discrete energy levels in the "shielded" channel. Measuring the pseudophase on the lattice can be used to determine the $\bar{K} N$ scattering length. This can be directly seen from the expression for the pseudophase, which depends on the real functions $H_{11}, H_{12}, H_{22}$. Extracting these from the data on energy levels, we then find the scattering length by using Eq. (3.2). A typical dependence of the energy levels on the variable $L$ in the coupled-channel case is shown in Fig. 3. Note that in the expression for the scattering length we need $H_{i j}(s)$ evaluated at threshold $s=s_{t}$. However, replacing $H_{i j}(s)$ by $H_{i j}\left(s_{t}\right)$ introduces a very small correction, since the effective range term proportional to $\left(s-s_{t}\right)$ is suppressed by $L^{-3}$ as compared to the leading order result. So, in the fit to the data, one may replace $H_{i j}(s)$ by their values at threshold. Using pseudodata generated from a K-matric model, we have checked that this method can indeed be used to determine $a_{11}$ from the pseudophase in the vicinity of the $K^{-} p$ threshold. To summarize this section, we have generalized Lüscher's algorithm for the extraction of the scattering length from the finite-volume energy spectrum measured on the lattice. The modified algorithm applies to the case when the scattering length is complex due to the 
presence of open channel(s) below threshold. In the case of $\bar{K} N$ scattering with total isospin $I=0$, the scattering length can be determined by measuring the volume dependence of the first excited level around the threshold energy.

\section{Chiral corrections to the Roper mass}

Understanding the (ir)regularities of the light quark baryon spectrum poses an important challenge for lattice QCD. In particular, the first even-parity excited state of the nucleon, the Roper $N^{*}(1440)$ (from here on called the Roper) is very intriguing - it is lighter than the first odd-parity nucleon excitation, the $S_{11}(1535)$, and also has a significant branching ratio into two pions. Recent lattice studies have not offered a clear picture about the nucleon resonance spectrum. In particular, in Ref. [19] an indication of a rapid cross over of the first positive and negative excited nucleon states close to the chiral limit was reported - so far not seen in other simulations at higher quark masses. Note also that so far very simple chiral extrapolation functions have been employed in most approaches, e.g., a linear extrapolation in the quark masses, thus $\sim M_{\pi}^{2}$, was applied in [20]. It is therefore important to provide the lattice practitioners with improved chiral extrapolation functions. A complete one-loop representation for the pion mass dependence of the Roper mass was given in [21]. Since the Roper is the first even-parity excited state of the nucleon, the construction of the chiral SU(2) effective Lagrangian follows standard procedures, see e.g. [22]. The effective Lagrangian relevant for our calculation is (working in the isospin limit $m_{u}=m_{d}$ and neglecting electromagnetism)

$$
\begin{aligned}
\mathscr{L} & =\mathscr{L}_{0}+\mathscr{L}_{R}+\mathscr{L}_{N R} \\
\mathscr{L}_{0} & =i \bar{N} \gamma_{\mu} D^{\mu} N-m_{N} \bar{N} N+i \bar{R} \gamma_{\mu} D^{\mu} R-m_{R} \bar{R} R \\
\mathscr{L}_{R}^{(1)} & =\frac{1}{2} g_{R} \bar{R} \gamma_{\mu} \gamma_{5} u^{\mu} R, \mathscr{L}_{N R}^{(1)}=\frac{g_{N R}}{2} \bar{R} \gamma_{\mu} \gamma_{5} u^{\mu} N+\text { h.c. }, \\
\mathscr{L}_{R}^{(2)} & =c_{1}^{*}\left\langle\chi_{+}\right\rangle \bar{R} R-\frac{c_{2}^{*}}{8 m_{R}^{2}} \bar{R}\left(\left\langle u_{\mu} u_{v}\right\rangle\left\{D^{\mu}, D^{v}\right\}+\text { h.c. }\right) R+\frac{c_{3}^{*}}{2}\left\langle u_{\mu} u^{\mu}\right\rangle \bar{R} R \\
\mathscr{L}_{R}^{(4)} & =-\frac{e_{1}^{*}}{16}\left\langle\chi_{+}\right\rangle^{2} \bar{R} R
\end{aligned}
$$

where $N, R$ are nucleon and Roper fields, respectively, and $m_{N}, m_{R}$ the corresponding baryon masses in the chiral limit. The pion fields are collected in $u_{\mu}=-\partial_{\mu} \pi / F_{\pi}+\mathscr{O}\left(\pi^{3}\right) . D_{\mu}$ is the chiral covariant derivative, for our purpose we can set $D_{\mu}=\partial_{\mu}$, see e.g. [22] for definitions. Further, $\chi_{+}$ is proportional to the pion mass and induces explicit chiral symmetry breaking, and \langle\rangle denotes the trace in flavor space. The dimension two and four LECs $c_{i}^{*}$ and $e_{i}^{*}$ correspond to the $c_{i}$ and $e_{i}$ of the effective chiral pion-nucleon Lagrangian [22]. The pion-Roper coupling is given to leading chiral order by $\mathscr{L}_{R}^{(1)}$, with a coupling $g_{R}$. This coupling is bounded by the nucleon axial coupling, $\left|g_{R}\right|<\left|g_{A}\right|$, in what follows we use $g_{R}=1$. The leading interaction piece between nucleons and the Roper is given by $\mathscr{L}_{N R}^{(1)}$. The coupling $g_{N R}$ can be determined from the strong decays of the Roper resonance, its actual value is $g_{N R}=0.35$ using the Roper width extracted from the speed plot (and not from a Breit-Wigner fit). Further pion-Roper couplings are encoded in $\mathscr{L}_{R}^{(2)}$ and $\mathscr{L}_{R}^{(4)}$. To analyze the real part of the Roper self-energy, one has to calculate a) tree graphs with insertion $\sim c_{1}^{*}, e_{1}^{*}$, self-energy diagrams with intermediate b) nucleon and c) Roper states and d) tadpoles 


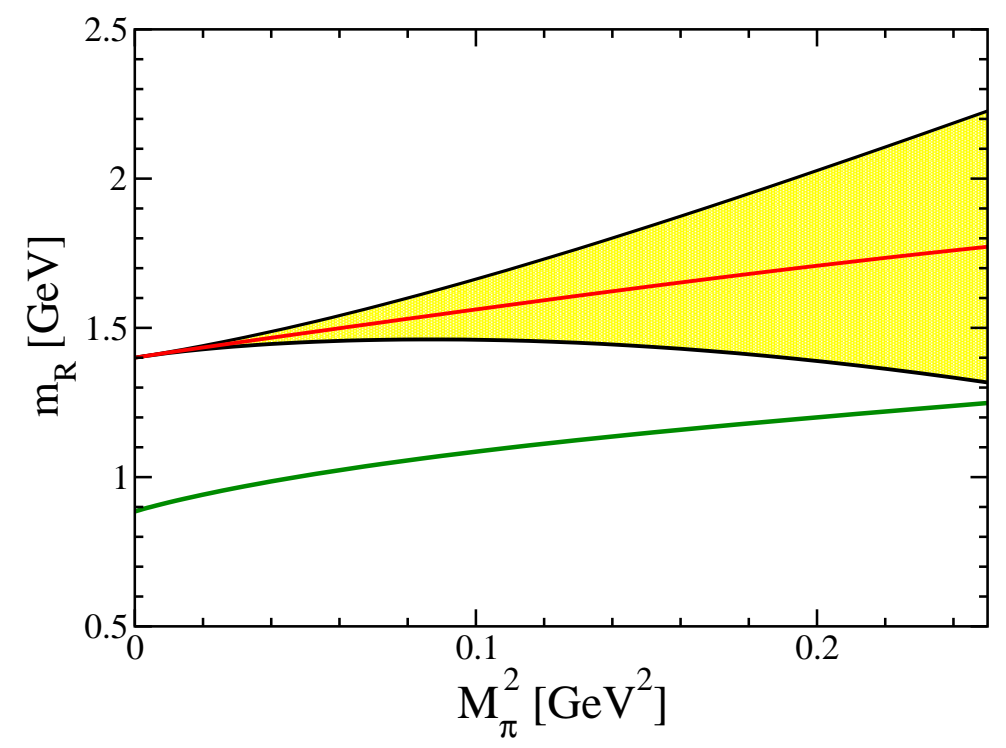

Figure 4: Quark mass dependence of the Roper mass for different parameter sets. The solid curve gives the result for the central values of the LECs, the hatched area corresponds to variations of these LECs within natural bounds. The dotted curve represents the quark mass dependence of the nucleon, see Ref. [23]. The values of the corresponding LECs are: $c_{1}=-0.9, c_{2}=3.2, c_{3}=-3.45, e_{1}=-1.0$.

with vertices from $\mathscr{L}_{R}^{(2)}$. In fact, the graphs of type b) require a modification of the regularization scheme due to the appearance of the two large mass scales $m_{N}$ and $m_{R}$. The solution to this problem - assuming $m_{N}^{2} / m_{R}^{2} \ll 1$ (in nature, this ratio is $\simeq 1 / 2.4$ ) - is described in [21]. As discussed in that paper, the LECs $c_{i}^{*}$ and $e_{i}^{*}$ can be bounded assuming naturalness and by direct comparison with the corresponding pion-nucleon couplings: $\left|c_{1}^{*}\right| \lesssim 0.5 \mathrm{GeV}^{-1},\left|c_{2,3}^{*}\right| \lesssim 1.0 \mathrm{GeV}^{-1}$ and $\left|e_{1}^{*}\right| \lesssim 0.5 \mathrm{GeV}^{-3}$. In Fig. 4 an estimated range for the pion mass dependence of the Roper mass is presented by taking the extreme values for $c_{2,3}^{*}$ and $e_{1}^{*}$, while keeping $c_{1}^{*}=-0.5 \mathrm{GeV}^{-1}, g_{N R}=0.35, g_{R}=1$ fixed. The masses of the baryons in the chiral limit are taken to be $m_{N}=0.885 \mathrm{GeV}$ [23] and $m_{R}=1.4 \mathrm{GeV}$, respectively. The upper solid curve is obtained by setting the couplings $c_{2,3}^{*}, e_{1}^{*}$ all to zero, and exhibits up to an offset a similar quark mass dependence as the nucleon result (lower solid curve, taken from Ref. [23]). It should be emphasized, however, that the one-loop formula cannot be trusted for pion masses much beyond $350 \mathrm{MeV}$. No sharp decrease of the Roper mass for small pion masses is observed for natural values of the couplings. Note that the important $\Delta \pi$ and $N \pi \pi$ channels are effectively included through the dimension two and four contact interactions, still it would be worthwhile to extend these considerations including the delta explicitely. Note further that the formalism developed in [21] is in general suited to study systems with two heavy mass scales in addition to a light mass scale. In this sense, it can be applied to other resonances as well, such as the $S_{11}(1535)$. In this case, however, an SU(3) calculation is necessary due to the important $\eta N$ decay channel.

\section{Acknowledgements}

I thank all my collaborators for sharing their insights into the topics discussed here and the 
orgainzers for a job well done. This work is supported in part by the BMBF under contract No. 06BN411, by the DFG (SFB/TR 16 "Subnuclear Structure of Matter"), by the Helmholtz Association through funds provided to the virtual institute "Spin and strong QCD" (VH-VI-231), and by the EU Integrated Infrastructure Initiative Hadron Physics Project under contract number RII3-CT2004-506078.

\section{References}

[1] V. Bernard, Prog. Part. Nucl. Phys. 60 (2008) 82 [arXiv:0706.0312 [hep-ph]].

[2] S. R. Sharpe, arXiv:hep-lat/0607016.

[3] C. Gattringer, arXiv:0711.0622 [hep-lat].

[4] J. Bulava et al., AIP Conf. Proc. 947 (2007) 77 [arXiv:0708.2072 [hep-lat]].

[5] C. Morningstar, arXiv:0810.4448 [hep-lat].

[6] S. Aoki et al. [CP-PACS Collaboration], Phys. Rev. D 76 (2007) 094506 [arXiv:0708.3705 [hep-lat]].

[7] M. Göckeler, R. Horsley, Y. Nakamura, D. Pleiter, P. E. L. Rakow, G. Schierholz and J. Zanotti, arXiv:0810.5337 [hep-lat].

[8] E. Jenkins and A. V. Manohar, Phys. Lett. B 259 (1991) 353.

[9] T. R. Hemmert, B. R. Holstein and J. Kambor, J. Phys. G 24 (1998) 1831 [arXiv:hep-ph/9712496].

[10] V. Bernard, U.-G. Meißner and A. Rusetsky, Nucl. Phys. B 788 (2008) 1 [arXiv:hep-lat/0702012].

[11] V. Bernard, D. Hoja, U.-G. Meißner and A. Rusetsky, arXiv:0902.2346 [hep-lat].

[12] C. Alexandrou et al. [European Twisted Mass Collaboration], Phys. Rev. D 78 (2008) 014509 [arXiv:0803.3190 [hep-lat]].

[13] A. Walker-Loud and J. M. S. Wu, Phys. Rev. D 72 (2005) 014506 [arXiv:hep-lat/0504001].

[14] B. C. Tiburzi, Nucl. Phys. A 761 (2005) 232 [arXiv:hep-lat/0501020].

[15] V. Bernard, M. Lage, U.-G. Meißner and A. Rusetsky, JHEP 0808 (2008) 024 [arXiv:0806.4495 [hep-lat]].

[16] U.-G. Meißner, U. Raha and A. Rusetsky, Eur. Phys. J. C 35 (2004) 349 [arXiv:hep-ph/0402261].

[17] M. Lage, U.-G. Meißner and A. Rusetsky, in preparation.

[18] S. He, X. Feng and C. Liu, JHEP 0507 (2005) 011 [arXiv:hep-lat/0504019]; C. Liu, X. Feng and S. He, Int. J. Mod. Phys. A 21 (2006) 847 [arXiv:hep-lat/0508022].

[19] N. Mathur et al., Phys. Lett. B 605 (2005) 137 [arXiv:hep-ph/0306199].

[20] T. Burch, C. Gattringer, L. Y. Glozman, C. Hagen, D. Hierl, C. B. Lang and A. Schäfer, Phys. Rev. D 74 (2006) 014504 [arXiv:hep-lat/0604019].

[21] B. Borasoy, P. C. Bruns, U.-G. Meißner and R. Lewis, Phys. Lett. B 641 (2006) 294 [arXiv:hep-lat/0608001].

[22] N. Fettes, U.-G. Meißner, M. Mojžiš and S. Steininger, Annals Phys. 283 (2000) 273 [Erratum-ibid. 288 (2001) 249] [arXiv:hep-ph/0001308].

[23] V. Bernard, T. R. Hemmert and U.-G. Meißner, Nucl. Phys. A 732 (2004) 149 [arXiv:hep-ph/0307115]. 